\begin{tabular}{|c|l|}
\hline Title & Experimental test of tight state independent preparation uncertainty relations for qubits \\
\hline Author(s) & Sponar, Stephan; Danner, A rmin; Obigane, Kazuma; Hack, Simon; Hasegawa, Yuji \\
\hline Citation & $\begin{array}{l}\text { Physical Review A, 102(4), 042204 } \\
\text { https://doi.org/10.1103/PhysRevA.102.042204 }\end{array}$ \\
\hline Issue Date & 2020-10-06 \\
\hline Doc URL & http://hdl.handle.net/2115/79765 \\
\hline Rights & O[2020] A merican Physical Society \\
\hline Type & article \\
\hline File Information & PhysRevA.102.042204.pdf \\
\hline
\end{tabular}

Instructions for use 


\title{
Experimental test of tight state-independent preparation uncertainty relations for qubits
}

\author{
Stephan Sponar $\odot,{ }^{1, *}$ Armin Danner $\odot,{ }^{1}$ Kazuma Obigane, ${ }^{2}$ Simon Hack $\odot,{ }^{1}$ and Yuji Hasegawa $\odot^{1,2, \dagger}$ \\ ${ }^{1}$ Atominstitut, TU Wien, Stadionallee 2, 1020 Vienna, Austria \\ ${ }^{2}$ Department of Applied Physics, Hokkaido University, Kita-ku, Sapporo 060-8628, Japan
}

(Received 3 February 2020; revised 4 September 2020; accepted 9 September 2020; published 6 October 2020)

\begin{abstract}
The well-known Robertson-Schrödinger uncertainty relations miss an irreducible lower bound. This is widely attributed to the lower bound's state dependence. Therefore, Abbott et al. introduced a general approach to derive tight state-independent uncertainty relations for qubit measurements [Mathematics 4, 8 (2016)]. The relations are expressed in two measures of uncertainty, which are standard deviation and entropy, both functions of the expectation value. Here, we present a neutron polarimetric test of the tight state-independent preparation uncertainty relations for orthogonal, as well as nonorthogonal, Pauli spin observables. The final results, obtained with pure and mixed spin states, reproduce the theoretical predictions clearly for arbitrary initial states of variable degree of polarization.
\end{abstract}

DOI: 10.1103/PhysRevA.102.042204

\section{INTRODUCTION}

The impossibility of assigning definite values to incompatible observables is a fundamental feature of quantum mechanics. It manifests in the impracticality of preparing quantum states that simultaneously have precise values of position $Q$ and momentum $P$. This is expressed in the wellknown position-momentum uncertainty relation $\Delta Q \Delta P \geqslant$ $\frac{\hbar}{2}$, which sets a lower bound on the product of standard deviations of the position and momentum observables. The position-momentum uncertainty relation was generally proved from basic principles of quantum mechanics by Kennard in 1927 [1], following Heisenberg's introduction of the uncertainty principle illustrated by the famous $\gamma$-ray microscope Gedankenexperiment [2]. However, the $\gamma$-ray microscope sets a lower bound for the product of the measurement error and the disturbance in a joint measurement of position $Q$ and momentum $P$ on a single particle. Hence, the position-momentum uncertainty relation in terms of standard deviations quantifies how precise, with respect to the observables of interest, a state can be prepared, rather than the ability to jointly measure them.

In 1929 Robertson generalized the uncertainty relation to arbitrary pairs of incompatible (i.e., noncommuting) observables $A$ and $B$ as

$$
\triangle A \Delta B \geqslant \frac{1}{2}|\langle\psi|[A, B]| \psi\rangle|,
$$

for any state $|\psi\rangle$, where $[A, B]$ represents the commutator $[A, B]=A B-B A$ and the standard deviation of an observable $X$ is defined as $(\Delta X)^{2}=\left\langle\psi\left|X^{2}\right| \psi\right\rangle-\langle\psi|X| \psi\rangle^{2}$ [3]. However, Robertson's uncertainty relation turned out to follow from a slightly stronger inequality namely the Schrödinger

\footnotetext{
*stephan.sponar@tuwien.ac.at

†yuji.hasegawa@tuwien.ac.at
}

uncertainty relation [4], given by

$$
\begin{aligned}
(\Delta A)^{2}(\Delta B)^{2} \geqslant & \left|\frac{1}{2}\langle\psi|\{A, B\}| \psi\rangle-\langle\psi|A| \psi\rangle\langle\psi|B| \psi\rangle\right|^{2} \\
& +\frac{1}{2}|\langle\psi|[A, B]| \psi\rangle|^{2},
\end{aligned}
$$

where the anticommutator $\{A, B\}=A B+B A$ is used. Here, the right-hand side (RHS) of Eq. (2) yields a tighter bound than Eq. (1), but is not necessarily saturated. A peculiarity of Robertson's and Schrödinger's uncertainty relations is the fact that the right-hand sides of Eqs. (1) and (2) are also state dependent, even though the statistics of both observables are independently accessible and undisturbed after state preparation.

Note that Kennard's, Robertson's, and Schrödinger's uncertainty relations all express a quantitative statement about the measurement statistics for $A$ and $B$ of different ensembles that are obtained separately (many times) on identically prepared quantum systems; this is the reason why such relations are usually referred to as preparation uncertainty relations. They propose fundamental limits on the measurement statistics for any state preparation.

The fact that in the case of preparation uncertainty relations the measurements are performed on different ensembles is in total contrast to Heisenberg's original discussion of his uncertainty principle, which addresses the inability to jointly (simultaneously or sequentially) measure incompatible observables with arbitrary accuracy, which is described by measurement uncertainty relations. Consequently, uncertainty relations have a long history of being misinterpreted as exclusive statements about joint measurements.

In recent years measurement uncertainty relations, as originally proposed by Heisenberg [2], have received renewed attention. One particular challenge here is the fact that measurement uncertainties are subtle to define, because no experimental evidence of the target observable is available between preparation and measurement. A detailed experimental investigation of this issue can be found in [5]. New uncertainty 
measures and uncertainty relations for error and disturbance have been proposed [6,7], refined [8,9], and experimentally tested in neutronic [10-15] and photonic [16-21] systems: In Ozawa's operator-based approach [6], or operator formalism, the measurement process is described by an indirect measurement model, introduced in [22]. Here the object system is coupled to a probe system by a unitary operator acting on the composite object-probe system. Then, the measurement error is defined as root-mean-square deviation between target observable before and meter observable after the measurement interaction. Hence, the operator formalism defines correlations between the measurement outcome and the target observable. Note that the state dependence in this model is necessary, because the value of the observable is (partially) determined by the input state. The disturbance induced on another observable by the first measurement is given by the root-mean-square difference between that observable before and after the measurement interaction.

Alternative approaches, for instance Busch, Lathi, and Werner's operational approach [7], aim to avoid the problem of state dependency by evaluating error and disturbance from differences between output probability distributions [7,23]. Therefore, single measurements (reference or control measurements) of the target observables are performed first. In a second step, a successive measurement of the two target observables is carried out. The marginal distributions of the joint probability distribution obtained from the successive measurement are compared with the output statistics of the single measurements. However, it should be emphasized here that such an approach is insensitive to certain types of errors, which has been pointed out in [24] (see [13] for an experimental comparison of these two approaches). In general it is difficult to avoid state dependence in measurement uncertainties. However, there continues to be some debate as to the appropriate measure of measurement (in)accuracy and of disturbance [6-8,10,11,13,16,18-21,23,25-30].

This recent interest in measurement uncertainty relations revealed that the well-known Robertson-Schrödinger uncertainty relation lacks an irreducible or state-independent lower bound of the RHS of Eq. (1). Owing to this fact the lower bound on the right-hand sides of Eqs. (1) and (2) may become zero for certain states, even for noncommuting $A$ and $B$, as is the case for instance for neutron spins. A solution to this problem was already proposed by Deutsch in the 1980s, who looked for a theorem of linear algebra in the form $\mathcal{U}(A, B, \psi) \geqslant \mathcal{B}(A, B)$, that is, a state-independent relation, and furthermore suggested to use (Shannon) entropy as measure [31]. Note that Heisenberg's (more precisely Kennard's) inequality $\Delta Q \Delta P \geqslant \frac{\hbar}{2}$ has that form, but its generalizations Eqs. (1) and (2) do not. Common to all entropic uncertainty relations is the peculiarity of setting bounds on the sum of the entropies of $A$ and $B$ rather than on the product. Deutsch's original entropic preparation uncertainty relation reads

$$
H(A)+H(B) \geqslant-2 \log _{2}\left(\frac{1+c}{2}\right),
$$

where $c=\max _{i, j}\left|\left\langle a_{i} \mid b_{j}\right\rangle\right|$ is the maximum overlap between the eigenvectors $\left|a_{i}\right\rangle$ and $\left|b_{j}\right\rangle$ of observables $A$ and $B$, respectively. Then the Shannon entropy $H(A)=$ $\sum_{i} \operatorname{Tr}\left[\rho P_{i}\right] \log _{2}\left(\operatorname{Tr}\left[\rho P_{i}\right]\right)$, with $P_{i}$ being a projector from the spectral decomposition of the observable $A$, given by $A=$ $\sum_{i} a_{i} P_{i}$, provides a measure of uncertainty for the observable $A$ in the state $\rho$. Deutsch's relation from Eq. (3) was later improved by Maassen and Uffink [32], following a conjecture of Kraus [33], as

$$
H(A)+H(B) \geqslant-2 \log _{2} c .
$$

Even better, but still not tight bounds for the sub-optimal relation in Eqs. (4) and (3) are derived in [34-37].

More recently, entropic uncertainty relations have been extended to include the case of quantum memories [38,39]. Uncertainty relations are often a powerful tool to witness entanglement. This is due to the fact that entanglement criteria based on uncertainties require state independence because the local state of an entangled system cannot be defined. State-independent uncertainties have been introduced as entanglement witnesses in [40] and [41]. These recent developments clearly indicate that uncertainty relations, in fact both preparation and measurement uncertainty relations, are an active field of research, where much of the theory is still being developed, as can be seen from publications such as [42].

\section{THEORETICAL FRAMEWORK}

The growth in the numbers of studies, both theoretical and experimental, in measurement uncertainty relations has prompted renewed interest in the possibility of stateindependent preparation uncertainty relations also for the standard deviations of observables, not only for entropic relations. In 2014 Busch et al. [23] proposed two stateindependent uncertainty relations for qubits, more precisely for arbitrary Pauli observables, denoted as $A=\vec{a} \cdot \vec{\sigma}$ and $B=$ $\vec{b} \cdot \vec{\sigma}$. These additive versions of a preparation uncertainty relations are expressed as a sum, instead of a product, of two standard deviations as

$$
\begin{aligned}
\Delta A+\Delta B & \geqslant|\vec{a} \times \vec{b}|, \\
(\Delta A)^{2}+(\Delta B)^{2} & \geqslant 1-|\vec{a} \cdot \vec{b}| .
\end{aligned}
$$

Although these state-independent relations can be saturated by certain states, they are not tight in general. The following year, Abbott and Branciard proposed an approach for deriving tight state-independent and partially state-dependent (that is depending on the mixing parameter $r$ of nonpure states) uncertainty relations for qubit measurements that completely characterize the obtainable uncertainty values reported in [43]. Their equivalent relations in terms of expectation values, standard deviations and entropies can more generally be transformed into other relations, in terms of any measure of uncertainty that can be written as a function of the expectation value. Any pair of Pauli observables $A=\vec{a} \cdot \vec{\sigma}$ and $B=\vec{b} \cdot \vec{\sigma}$, with $\vec{\sigma}=\left(\sigma_{x}, \sigma_{y}, \sigma_{z}\right)^{T}$ and an arbitrary quantum state $\rho=\frac{1}{2}(\mathbb{1}+\vec{r} \cdot \vec{\sigma})$ satisfies the condition

$$
\begin{aligned}
|\langle A\rangle \vec{a}-\langle B\rangle \vec{b}|^{2} & =\langle A\rangle^{2}+\langle B\rangle^{2}-2(\vec{a} \cdot \vec{b})\langle A\rangle\langle B\rangle \\
& \leqslant\left(1-(\vec{a} \cdot \vec{b})^{2}\right)|\vec{r}|^{2} \leqslant 1-(\vec{a} \cdot \vec{b})^{2}=|\vec{a} \times \vec{b}|^{2},
\end{aligned}
$$


(a)

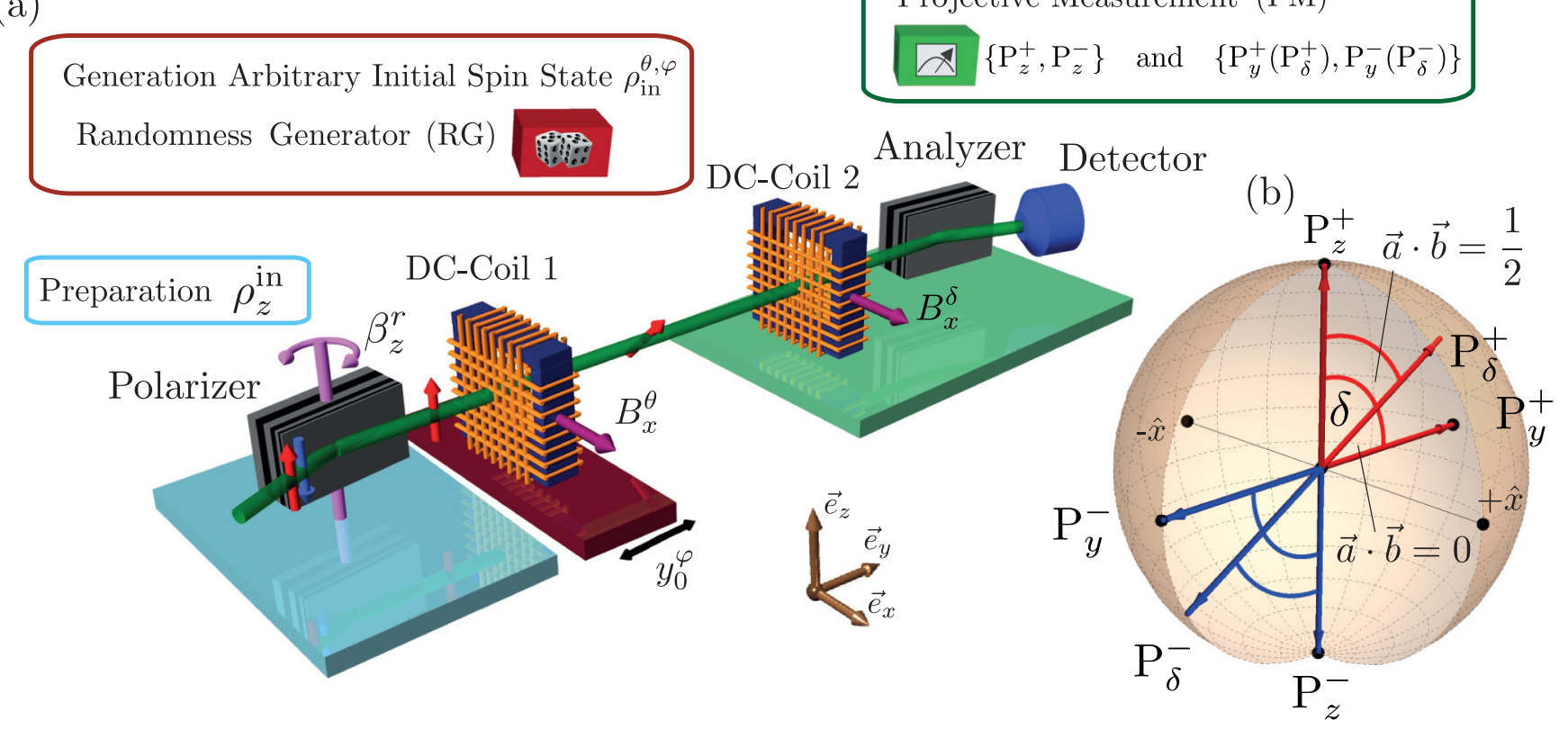

FIG. 1. (a) Experimental setup for determining the tight preparation uncertainty relation for neutron spins. Blue: preparation of incident state $\rho_{z}^{\text {in }}$. Ruby: the initial state $\rho_{\text {in }}^{\theta, \varphi}$ is chosen by the randomness generator (RG) and generated by DC coil 1, where polar angle $\theta$ is dependent on the static magnetic field $B_{x}^{\mathrm{RG}}$ and azimuthal angle $\varphi$ on the position $y_{0}$. Green: the projectors $P_{z}^{+}, P_{z}^{-}, P_{y}^{+}\left(P_{\delta}^{+}\right)$, and $P_{y}^{-}\left(P_{\delta}^{-}\right)$. are realized by the action of the supermirror (analyzer), while applying the respective magnetic fields in DC coil 2. (b) Bloch sphere description of projectors $P_{z}^{+}, P_{y}^{+}, P_{\delta}^{+}$and $P_{z}^{-}, P_{y}^{-}, P_{\delta}^{-}$.

where $\langle X\rangle$ denots the expectation value of $X$, that is, $\langle\psi|X| \psi\rangle$ for pure states $|\psi\rangle$, or $\operatorname{Tr}(\rho X)$ for mixed states $\rho$. Standard deviation $\Delta X$ and expectation value $\langle X\rangle$ are connected via

$$
(\Delta X)^{2}=1-\langle X\rangle^{2} \text { and }\langle X\rangle= \pm \sqrt{1-(\Delta X)^{2}},
$$

since every Pauli operator $X$ satisfies $X^{2}=11$. Hence, the tight state-independent uncertainty relation, given in Eq. (6), can be rewritten in terms of standard deviations as

$$
\begin{aligned}
& (\Delta A)^{2}+(\Delta B)^{2}+2|\vec{a} \cdot \vec{b}| \sqrt{1-(\Delta A)^{2}} \sqrt{1-(\Delta B)^{2}} \\
& \geqslant 2-\left[1-(\vec{a} \cdot \vec{b})^{2}\right]|\vec{r}|^{2} \geqslant 1+(\vec{a} \cdot \vec{b})^{2} .
\end{aligned}
$$

Note that Eq. (8) coincides with Eq. (5b) for orthogonal observables, i.e., $\vec{a} \cdot \vec{b}=0$. An alternative approach studying uncertainty relations that give lower bounds to the sum of standard deviations was recently presented in [44].

In the case of qubits, the Shannon entropy of a Pauli observable $X, H(X)$, can be directly expressed in terms of the expectation value $\langle X\rangle$, namely

$$
H(X)=h_{2}\left(\frac{1+\langle X\rangle}{2}\right)=h_{2}\left(\frac{1-\langle X\rangle}{2}\right),
$$

where $h_{2}$ is the binary entropy function defined as

$$
h_{2}(p)=-p \log _{2} p-(1-p) \log _{2}(1-p),
$$

or $\langle X\rangle= \pm f(H(X))$ with $f(x):=1-2 h_{2}^{-1}(x)$, where $h_{2}^{-1}$ denotes the inverse function of $h_{2}$. Then one obtains the following tight relation for two Pauli observables $A$ and $B$ :

$$
\begin{aligned}
& f(H(A))^{2}+f(H(B))^{2}-2|\vec{a} \cdot \vec{b}| f(H(A)) f(H(B)) \\
& \quad \leqslant\left[1-(\vec{a} \cdot \vec{b})^{2}\right]|\vec{r}|^{2} \leqslant 1-(\vec{a} \cdot \vec{b})^{2} .
\end{aligned}
$$

Note that the uncertainty relations in terms of standard deviations and entropy, which are given by Eqs. (8) and (11), are tight (state-independent) relations.

\section{EXPERIMENTAL SETUP AND PROCEDURE}

In this paper, we present a neutron optical test of the tight state-independent preparations uncertainties described by Eqs. (6), (8), and (11). The experiment was carried out at the polarimeter instrument NepTUn (NEutron Polarimeter TU wieN), located at the tangential beam port of the $250 \mathrm{~kW}$ TRIGA research reactor at the Atominstitut, TU Wien, in Vienna, Austria. A schematic illustration of the experimental setup is depicted in Fig. 1. An incoming monochromatic neutron beam with mean wavelength $\lambda \simeq 2.02 \AA(\Delta \lambda / \lambda \simeq 0.02)$ is polarized along the vertical $(+z)$ direction by refraction from a tunable CoTi multilayer array, henceforth referred to as supermirror. The incident (mixed) state is given by $\rho_{z}^{\text {in }}=\frac{1}{2}\left(\mathbb{1}+r \sigma_{z}\right)$, where $r=1$ corresponds to the pure state $|+z\rangle$. The mixing parameter $r$ is is adjusted by the incident angle $\beta_{z}^{r}$ between the supermirror and the neutron beam, in the required parameter region. Experimentally, initial degrees of polarizations between $r_{\min }=0.83(1)$ and $r_{\max }=0.99(1)$ were achieved. To avoid depolarization, the setup is covered by a $13 \mathrm{G}$ guide field in the vertical $(+z)$ direction (not depicted in Fig. 1). The initial states are chosen by a classical randomness generator (RG) and prepared by direct current (DC) coil 1, which generates a static magnetic field $B_{x}^{\theta}$ pointing in the $x$ direction. The magnetic field induces a unitary Larmor precession $U_{\mathrm{DC}}=e^{i \theta \sigma_{x}}$ by an angle $\theta$ inside the coil, expressed as

$$
\rho_{\text {in }}^{\theta}=U_{\mathrm{DC}}^{\dagger} \rho_{z}^{\mathrm{in}} U_{\mathrm{DC}} .
$$


The angle of rotation $\theta=\gamma B_{x}^{\theta} t$ is proportional to the magnetic field strength and the time $t$ of passage of the neutron through the coil, $\gamma$ being the gyromagnetic ratio $\gamma=-\frac{2|\mu|}{\hbar}$, where $\mu$ denotes the magnetic moment of the neutron. Since the transition time $t$ is constant, the polar angle $\theta$ of the initial spin state $\rho_{\text {in }}^{\theta}$ is entirely controlled by the electric current in the coil that generates the magnetic field $B_{x}^{\theta}$. The azimuthal angle $\varphi$ of the prepared initial state $\rho_{\text {in }}^{\theta, \varphi}$, given by

$$
\rho_{\mathrm{in}}^{\theta, \varphi}=U_{\mathrm{GF}}^{\dagger} U_{\mathrm{DC}}^{\dagger} \rho_{z}^{\mathrm{in}} U_{\mathrm{DC}} U_{\mathrm{GF}}
$$

and $U_{\mathrm{GF}}=e^{i \varphi \sigma_{z}}$, is induced by Larmor precession within the static magnetic guide field (GF). The respective angle is adjusted by the appropriate position $y_{0}^{\varphi}$ of DC coil 1 . Note that all randomly selected initial states lie on the boundary region of the respective tight uncertainty relation and belong to a subset of all possible states.

Our experimental test of the tight uncertainty relations Eqs. (6)-(11) is conducted for two fixed Pauli operators, given by $A=\vec{a} \cdot \vec{\sigma}$ and $B=\vec{b} \cdot \vec{\sigma}$, with (i) $\vec{a} \cdot \vec{b}=0$ and (ii) $\vec{a} \cdot \vec{b}=\frac{1}{2}$. For (i) we chose $\vec{a}=(0,0,1)^{T}$ and $\vec{b}=(0,1,0)^{T}$, thus the four projectors $P_{z}^{+}, P_{z}^{-}, P_{y}^{+}$, and $P_{y}^{-}$were measured for every randomly chosen initial state $\rho_{\text {in }}^{\theta, \varphi}$, resulting in an observed intensity denoted as $I=\operatorname{Tr}\left(\rho_{\text {in }}^{\theta, \varphi} P_{i}^{j}\right)$, with $i=z, y$ and $j=+,-$. The projectors are realized by the action of the supermirror (analyzer) while applying the respective magnetic fields in DC coil 2. Inside DC coil 2, the magnetic field $B_{x}^{\delta}$ induces spinor rotations of $\delta=0, \pi,+\frac{\pi}{2}$ and $\delta=-\frac{\pi}{2}$ about the $x$ axis, required for projective measurements along the $+z,-z,+y$, and $-y$ directions, respectively. Since all four projectors lie in the $y-z$ plane, the position of DC coil 2 remains unchanged. For (ii) $\vec{a} \cdot \vec{b}=\frac{1}{2}$, which corresponds to an angle of $\delta=60^{\circ}$ between $\vec{a}$ and $\vec{b}$, we have again $\vec{a}=$ $(0,0,1)^{T}$ but $\vec{b}=\left(0, \frac{\sqrt{3}}{2}, \frac{1}{2}\right)^{T}$ now. The respective projectors are denoted as $P_{z}^{+}, P_{z}^{-}, P_{\delta}^{+}$, and $P_{\delta}^{-}$, respectively.

\section{EXPERIMENTAL RESULTS}

\section{A. State-independent relations}

The lowest or state-independent bound, expressed by the very RHSs of Eqs. (6), (8), and (11), for expectation values, standard deviations and entropies, respectively, is saturated for pure initial states $(r=1)$, which will be studied first.

\section{Configuration $\vec{a} \cdot \vec{b}=0$}

Two Pauli observables $A=\vec{a} \cdot \vec{\sigma}$ and $B=\vec{b} \cdot \vec{\sigma}$, with $\vec{a}=$ $+\hat{z}$ and $\vec{b}=+\hat{y}$, yielding $\vec{a} \cdot \vec{b}=0$, are selected (see Fig. 2). Pure initial states, located on the great circle in the plane spanned by the observables' unit vectors $\vec{a}$ and $\vec{b}$, depicted in blue on the Bloch sphere in Fig. 2, form the lower bound of allowed values. However, the lower bound is not given by a closed curve in case of standard deviations and entropies. Therefore, additional initial states, indicated by the green arc [connection points (2)-(3)] and light green arc [connection points (1)-(3)] on the Bloch sphere, are required to close the boundary of all allowed values.

(i) Expectation values (EV): Equation (6) sets tight constraints on the allowed values for the expectation values $\langle A\rangle$

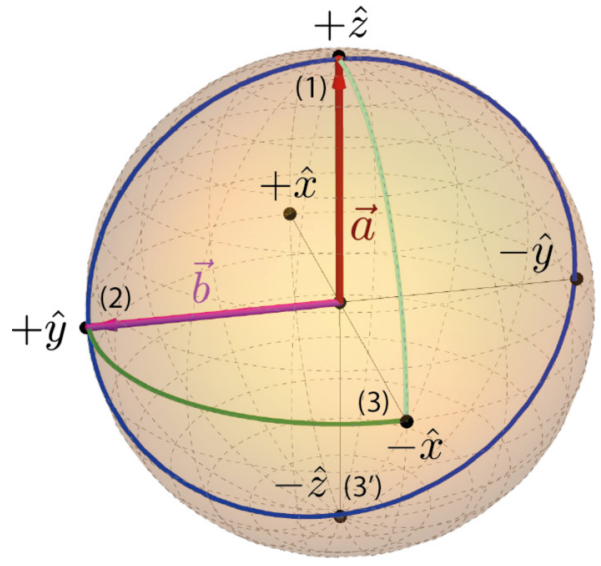

FIG. 2. Bloch sphere of initial states saturating boundaries of Eqs. (6), (8), and (11), for expectations values, standard deviations, and entropies, respectively, in the case $\vec{a} \cdot \vec{b}=0$.

and $\langle B\rangle$ which is experimentally tested with a set of randomly chosen initial states $\rho_{\text {in }}^{\theta}$. The state-independent bound of Eq. (6), given by $b_{\mathrm{EV}}=|\vec{a} \times \vec{b}|^{2}$, is saturated only by pure states, distributed on the great circle connecting north and south poles of the Bloch sphere via $+y$. This great circle, depicted in blue in Fig. 2, is embedded in the plane spanned by the observables' unit vectors $\vec{a}$ and $\vec{b}$ and parametrized by the polar angle $\theta \in[0,2 \pi]$ and $\varphi=\frac{\pi}{2}$. There is a one-to-one correspondence between the initial states' polar angle $\theta$ and the angle on the circle forming the boundary of allowed values for expectation values of $\langle A\rangle$ and $\langle B\rangle$, plotted in Fig. 3(a). Therefore, in the actual experiment the position of DC coil 1 remains fixed for this measurement. Starting at the north pole $(\theta=0)$, indicated as point (1) in Fig. 2, we have $\langle A\rangle=1$ and $\langle B\rangle=0$. At $\theta=\frac{\pi}{2}$ ( $+y$ direction), indicated by point (2), the situation reverses with $\langle A\rangle=0$ and $\langle B\rangle=1$. Closing the great circle on the Bloch sphere from $\theta=\frac{\pi}{2}$ to $\theta=2 \pi$ yields a closed curve for the boundary of all possible values of expectation values $\langle A\rangle$ and $\langle B\rangle$. Initial states outside the blue great circle, for instance states connecting points (2) and (3) - light green states in Fig. 2-are unbounded pure states, as seen from Fig. 3(a). At point (3) ( $-x$ direction) expectation values yield $\langle A\rangle=\langle B\rangle=0$, and are therefore found at the origin, which is the center of the region of allowed values for expectation values $\langle A\rangle$ and $\langle B\rangle$.

(ii) Standard deviations (SD): Since expectation value and standard deviation of Pauli observables are one-to-one related via $\Delta X=\sqrt{\langle X\rangle^{2}-1}$, the data obtained above are accordingly transformed to evaluate the tight state-independent preparation uncertainty relations as expressed in Eq. (8), with lower state-independent bound $b_{\mathrm{SD}}=1+(\vec{a} \cdot \vec{b})^{2}$. Unlike in the case of expectation values, pure states on the great circle in the $y-z$ plane saturate only the (state-independent) lower bound (curved boundary) but do not cover the entire region of allowed values for standard deviations $\Delta A$ and $\Delta B$, which can be seen in Fig. 3(b). At $\theta=0$ [point (1), $+z$ direction], standard deviation $\Delta B$ starts at maximal value (for $r=1$ this is $\Delta B=1$ ) and $\Delta A$ is minimal (for $r=1$ this is $\Delta A=0$ ). For increasing values of $\theta$ (while keeping $\varphi=\frac{\pi}{2}$ constant) $\Delta B$ decreases while $\Delta A$ increases. At $\theta=\frac{\pi}{2}$ [point (2), $+y$ 
(a)

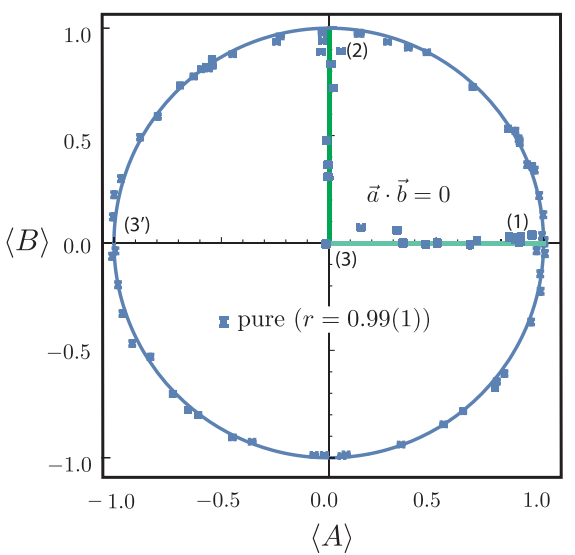

(b)

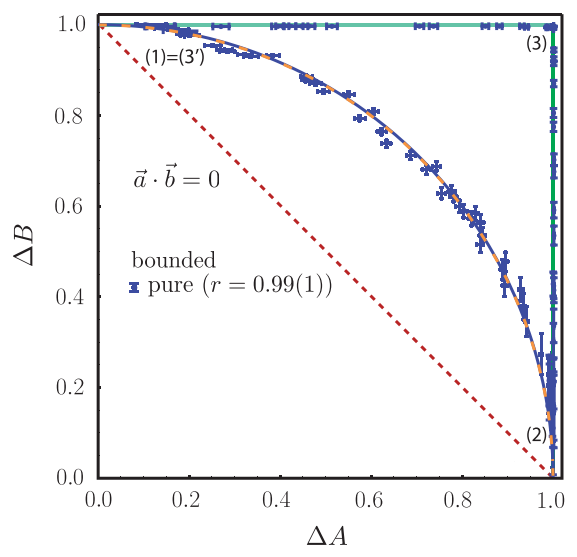

(c)

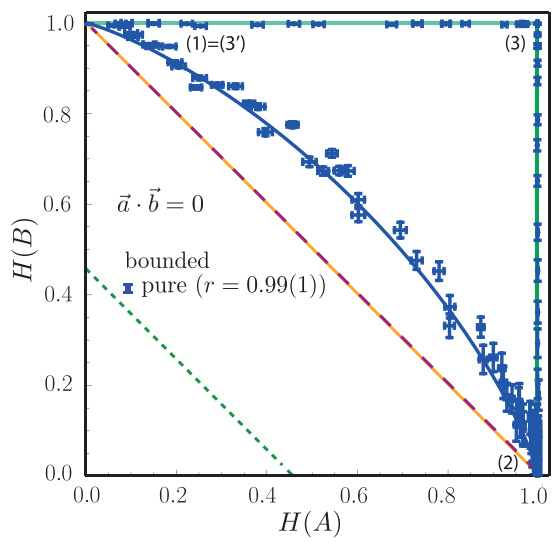

FIG. 3. Plots of the experimentally obtained values for Pauli observables $\vec{a} \cdot \vec{b}=0$, in terms of (a) expectation values $\langle A\rangle$, $\langle B\rangle$; (b) standard deviations $\Delta A, \Delta B$ [including bounds from Busch's Eqs. (5a) (dotted red line) and (5b) (dashed orange curve)]; and (c) entropies $H(A), H(B)$ [including bounds from Deutsch's Eq. (3) (dotted green line), from Maassen and Uffink (4) (dashed violet line), and from [34-37] (dashed beige line; same as dashed violet line)]. Blue curves [same as dashed orange curve in (b)] indicate the theoretic predictions of lower bounds from Eqs. (6), (8), and (11), for expectation values, standard deviations, and entropies, respectively. Dark green line [connecting points (1)-(3)] and light green line [connecting points (2)-(3)] represent the theoretic predictions for values of the corresponding initial states indicated by the respective color (points) on the Bloch sphere in Fig. 2.

direction) $\Delta B$ is minimal (for $r=1, \Delta B=0$ ) and $\Delta A$ is maximal (for $r=1, \Delta A=1$ ). In the interval $\theta \in\left[\frac{\pi}{2}, \pi\right]$ the reverse behavior is observed and at $(\theta, \varphi)=\left(\frac{\pi}{2}, \pi\right)$ [point (3), $-x$ direction], we have again $\Delta A=0$ and $\Delta B=1$ (as for $\theta=0)$. For $\theta \in[\pi, 2 \pi]$, the results of $\theta \in[0, \pi]$ are reproduced. The vertical boundary, corresponding to a constant (maximal) value of $\Delta A=1$, is obtained for initial states $\rho_{\text {in }}^{\theta, \varphi}$ with constant polar angle $\theta=\frac{\pi}{2}$ and randomly generated azimuthal angle $\varphi \in\left[\frac{\pi}{2}, \pi\right]$, these states are found on the dark green region of the equatorial plane of the Bloch sphere in Fig. 2. For $(\theta, \varphi)=\left(\frac{\pi}{2}, \pi\right)$, point (3), the upper right corner with $\Delta A=\Delta B=1$ is reached. For the horizontal boundary $(\Delta B=1), \varphi$ is kept constant at $\varphi=\pi$, while $\theta$ is randomly chosen from the interval $\left[0, \frac{\pi}{2}\right]$ (light green curve on the Bloch sphere), where for $\theta=0(+z$ direction) the boundary becomes a closed curve. Figure 3(b) also shows Busch's two additive versions of state-independent preparation uncertainty relations from Eq. (5a) (dotted red line) and Eq. (5b) (dashed orange curve). While the former is saturated only in points (1) and (2), the latter coincides with the bound from Eq. (8) (only for orthogonal observables $A$ and $B$ ).

(iii) Entropies: The approach presented in [43] for a tight state-independent uncertainty relations for qubits is based on the fact that in the case of Pauli observables the expectation value contains all information necessary to derive the uncertainty. Consequently, the uncertainty can also be expressed in terms of entropy $H$, which is also a function of the expectation value. The lower (state-independent) bound is consequently calculated as $b_{H}=1-(\vec{a} \cdot \vec{b})^{2}$. All arguments on the initial states saturating the boundary for standard deviations $\triangle A$ and $\Delta B$ also apply to entropies $H(A)$ and $H(B)$, which are plotted in Fig. 3(c). Figure 3(c) includes Deutsch's (suboptimal) bound from Eq. (3) (dotted green line) as well as the two bounds, in the case of orthogonal observables $A$ and $B$ being identical, from the Maasen-Uffink Eq. (4) (dashed violet line) and from [34-37] (dashed beige line), which are saturated only in points (1) and (2).

\section{Configuration $\vec{a} \cdot \vec{b}=\frac{1}{2}$}

Next, expectation values, standard deviations, and entropies for Pauli observables $A=\vec{a} \cdot \vec{\sigma}$ and $B=\vec{b} \cdot \vec{\sigma}$, with $\vec{a} \cdot \vec{b}=\frac{1}{2}$ (plotted in Fig. 4), which corresponds to a relative angle $\delta=60^{\circ}$ (see Bloch sphere in Fig. 5), are investigated.

(i) Expectation value: The obtained values for $\langle A\rangle$ and $\langle B\rangle$ now have an elliptical boundary, which is depicted in Fig. 4(a). For pure states with $\theta=0$ [point (1), $+z$ direction), neither of the two expectation vales $\langle A\rangle$ and $\langle B\rangle$ is zero, more precisely $\langle A\rangle=1$ and $\langle B\rangle=\frac{1}{2}$. For increasing values of $\theta$ (while keeping $\varphi=\frac{\pi}{2}$ constant) $\langle A\rangle=1$ decreases, while $\langle B\rangle=1$ increases, reaching a maximum of $\langle B\rangle=1$ (with $\langle A\rangle=\frac{1}{2}$ ) at $\theta=\frac{\pi}{3}=\delta$, that is the polar angle of unit vector $\vec{b}$, indicated by point (2) on the Bloch sphere in Fig. 5. In the interval $\theta \in$ $\left[\frac{\pi}{3}, \frac{\pi}{2}\right]$ both $\langle A\rangle$ and $\langle B\rangle$ are decreasing. At $\theta=\frac{\pi}{2}$ [point (3), $+y$ direction] $\langle A\rangle=0$ and $\langle B\rangle=0.87$. Polar angle $\theta=\frac{5 \pi}{6}$ yields $\langle B\rangle=0$ and $\langle A\rangle=-0.87$, at point $\left(4^{\prime}\right)$. A minimum for $\langle A\rangle$ is reached at $\theta=\pi$ [point $\left(5^{\prime}\right),-z$ direction] with $\langle A\rangle=-1$ (and $\langle B\rangle=-\frac{1}{2}$ ). In the interval $\theta \in[\pi, 2 \pi]$ the reverse behavior is observed. Initial states outside the blue great circle, for instance states connecting points (3) and (4), are unbounded pure states.

(ii) Standard deviations: Initial states that saturate the state-independent lower bound of Eq. (8) [curved boundaries from point (5) to point (3) in Fig. 4(b)] are located on the blue great circle in the $z-y$ plane of Fig. 4(a) with polar angle $\theta \in\left[-\frac{\pi}{6}, \frac{\pi}{2}\right]$. For pure states with $\theta=0$ [point (1), $+z$ direction] $\Delta A=0(\Delta B=0.87)$ is obtained. At $\theta=\frac{\pi}{3}=$ $\delta$, point (2), we have $\Delta B=0(\Delta A=0.87)$ and at $\theta=\frac{\pi}{2}$, point (3), $\Delta A=1$ and $\Delta B=\frac{1}{2}$. The vertical boundary, rep- 
(a)

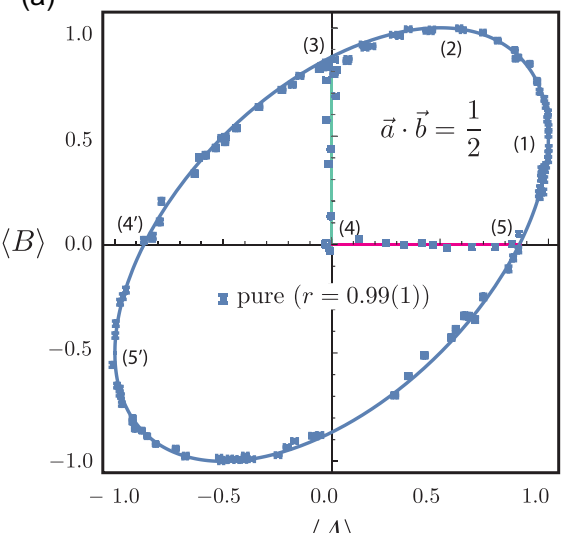

(b)

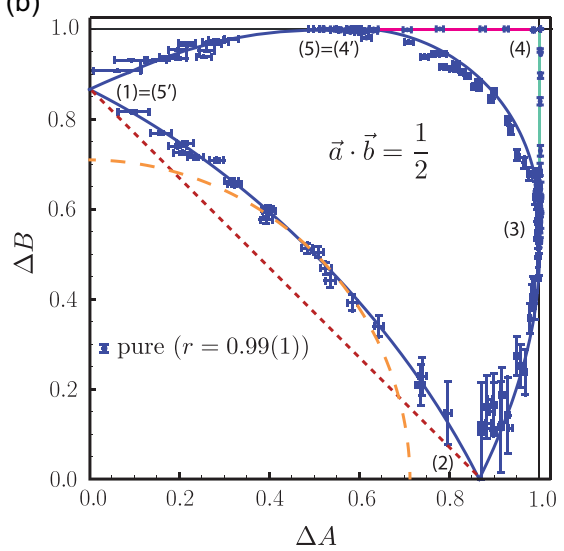

(c)

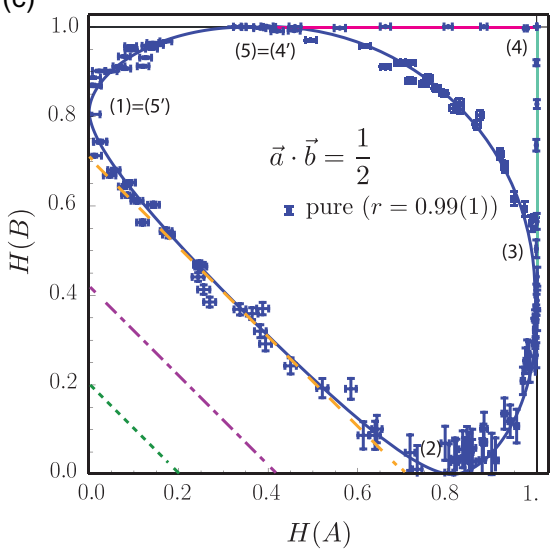

FIG. 4. Plots of the experimentally obtained values for Pauli observables $\vec{a} \cdot \vec{b}=\frac{1}{2}$, in terms of (a) expectation values $\langle A\rangle$, $\langle B\rangle$; (b) standard deviations $\Delta A, \Delta B$ [including bounds from Busch's Eqs (5a) (dotted red line) and (5b) (dashed orange curve)]; and (c) entropies $H(A), H(B)$ [including bounds from Deutsch's Eq. (3) (dotted green line), Maassen and Uffink (4) (dotted-dashed violet line), and from [34-37] (dashed beige line)]. Blue curves indicate the theoretic predictions of lower bounds from Eqs. (6), (8), and (11), for expectation values, standard deviations, and entropies, respectively. Light green line [connecting points (3)-(4)] and magenta line [connecting points (4)-(5)] represent the theoretic predictions for values of the corresponding initial states indicated by the respective color (points) on the Bloch sphere in Fig. 5 .

resented by points (3) to (4) is covered by initial states on the equatorial plane of the Bloch sphere with azimuthal angle $\varphi \in$ $\left[\frac{\pi}{2}, \pi\right]$ [light green line in Fig. 4(b)]. Initial states saturating the horizontal lower bound [magenta in Fig. 4(b)] are located on a great circle (magenta in Fig. 5) embedded in a plane perpendicular to $\vec{b}$. Here both polar angle $\theta$ and azimuthal angle $\varphi$ are varied, namely $\theta$ between $\frac{\pi}{2}$ and $-\frac{\pi}{6}$ and $\varphi$ between $\pi$ and $\frac{\pi}{2}$, before reaching point (4), thereby closing the boundary of allowed values for standard deviations $\Delta A$ and $\Delta B$. Initial states on the blue great circle in the $z-y$ plane of Fig. $5\left(\varphi=\frac{\pi}{2}\right)$ with $\theta>\frac{\pi}{2}$ are located inside the boundary (unbounded pure states), more precisely on the blue curve in Fig. 4(b) between point (3) and point $\left(4^{\prime}\right)$. At $\theta=\frac{5 \pi}{6}$, point $\left(4^{\prime}\right)$ is reached, yielding the same values as $\theta=-\frac{\pi}{6}$ of point (5). Busch's bound from Eq. (5a) (dotted red line) is saturated again only in points (1) and (2), whereas the bound from Eq. (5a) (dashed

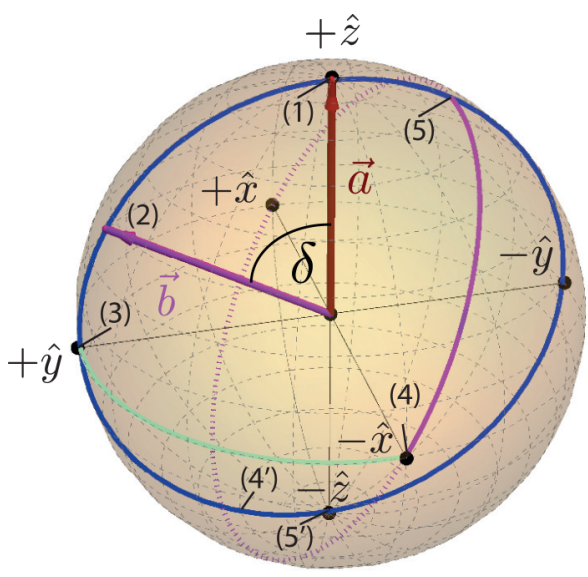

FIG. 5. Bloch sphere of initial states saturating boundaries of Eqs. (6), (8), and (11), for expectations values, standard deviations, and entropies, respectively, in the case $\vec{a} \cdot \vec{b}=\frac{1}{2}$. orange curve) touches the uncertainty region only in one point, namely $\left(\Delta(A),(\Delta(B))=\left(\sqrt{\frac{1-|\vec{a} \cdot \vec{b}|}{2}}, \sqrt{\frac{1-|\vec{a} \cdot \vec{b}|}{2}}\right)=\left(\frac{1}{2}, \frac{1}{2}\right)\right.$.

(iii) Entropies: Initial states saturating the boundary for entropies $H(A)$ and $H(B)$ are again the same as for standard deviations $\Delta A$ and $\Delta B$, which is plotted in Fig. 4(c). Values for entropies $H(A)$ and $H(B)$ in point $(i)$, denoted as $\{p(i) ; H(A), H(B)\}$, are given by $\{p(1) ; 0,0.81\},\{p(2) ; 0.81,0\},\{p(3) ; 1,0.35\},\{p(4) ; 1,1\}$, and $\{p(5) ; 0.35,1\}$. Deutsch's bound from Eq. (3) (dotted green line) and the bound from the Maasen-Uffink Eq. (4) (dotted-dashed violet line) are both suboptimal in the case of nonorthogonal observables $A$ and $B$. However, the bound from from [34-37] (dashed beige line) is saturated in a single point, that is found at $\left(H(A),(H(B))=\left(h_{2}\left(\frac{1+c}{2}\right), h_{2}\left(\frac{1+c}{2}\right)\right)=\right.$ $(0.345,0.345)$, where $c=\max _{i, j}\left|\left\langle a_{i} \mid b_{j}\right\rangle\right|$ is the maximum overlap between the eigenvectors $\left|a_{i}\right\rangle,\left|b_{j}\right\rangle$ of observables $A$ and $B$.

\section{B. Partially state-dependent relations 1. Expectation values}

As already discussed in Sec. IV A 1 , the state-independent bound of Eq. (6), given by $b_{\mathrm{EV}}=|\vec{a} \times \vec{b}|^{2}$, is saturated only by pure states, found on the surface of the $y$-z-plane on the Bloch sphere. The partially state-dependent lower bound, expressed as $b_{\mathrm{EV}}^{\prime}(r)=\left(1-(\vec{a} \cdot \vec{b})^{2}\right)|\vec{r}|^{2}$, is covered by mixed states located in the $y-z$ plane of the Bloch sphere, with respective degree of polarization $r$. For expectation values the lower bound of Eq. (6) is a closed curve representing the entire boundary of allowed values for $\langle A\rangle$ and $\langle B\rangle$, which can be seen in Figs. 6(a) and 6(b), for $\vec{a} \cdot \vec{b}=0$ and $\vec{a} \cdot \vec{b}=\frac{1}{2}$, respectively. The measurement is carried out for three initial degrees of polarization, which are tuned by the angle $\beta_{z}^{r}$ between the supermirror and the neutron beam, namely $r_{\min }=0.83(1)$, $r_{\text {mid }}=0.94(1)$, and $r_{\max }=0.99(1)$. The theoretical predictions for expectations vales $\langle A\rangle$ and $\langle B\rangle$, indicated by solid 
(a)

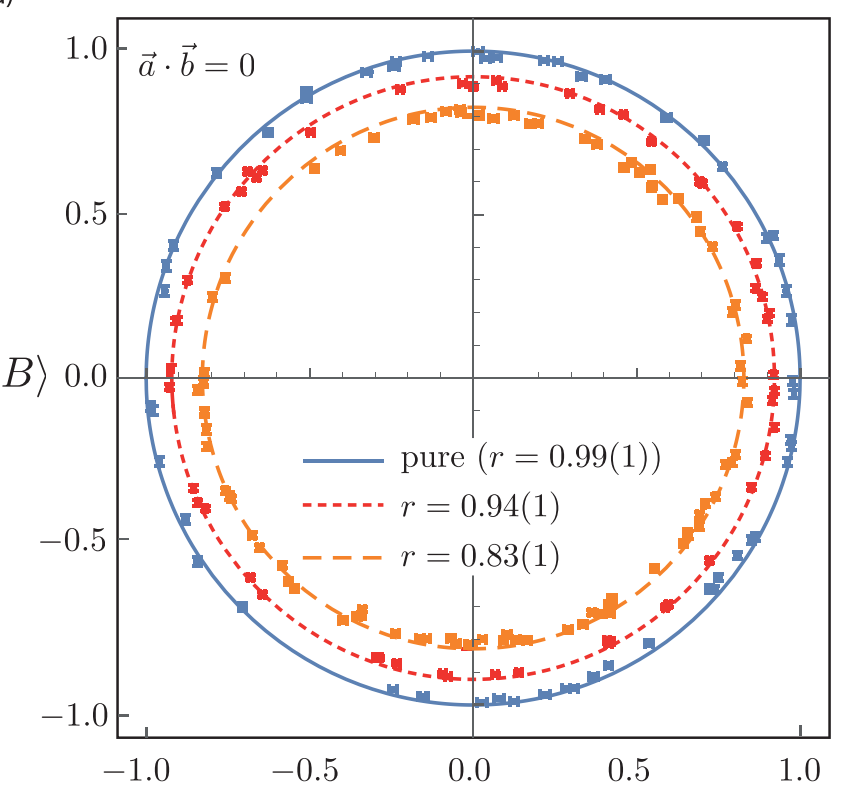

$\langle A\rangle$

(b)

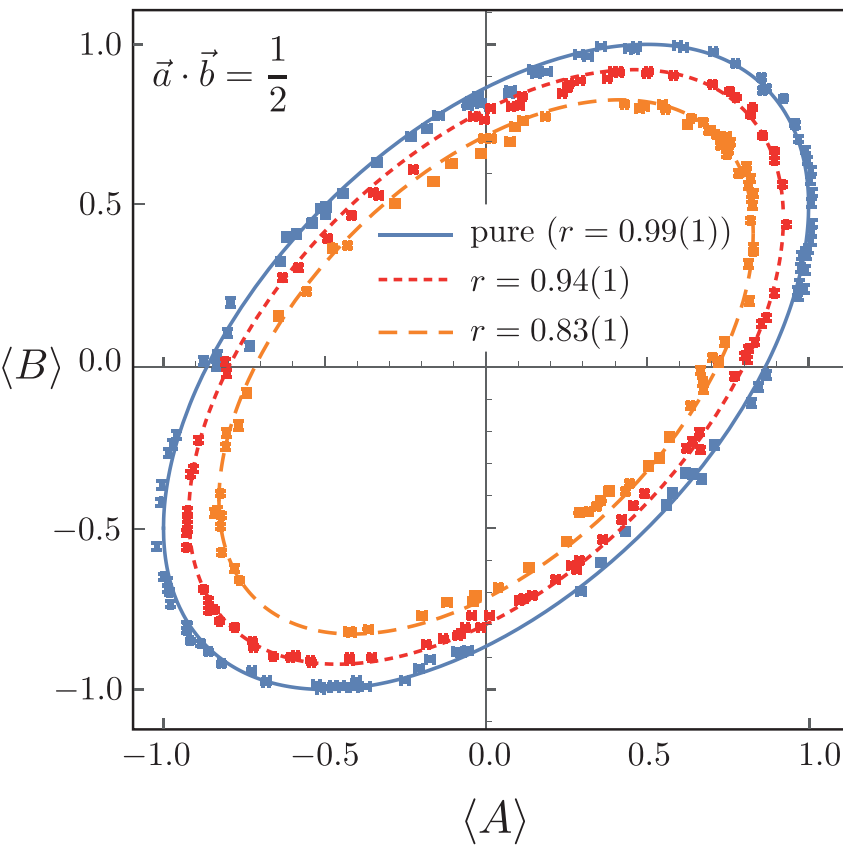

FIG. 6. Plots of the experimentally obtained values for expectation values $\langle A\rangle$ and $\langle B\rangle$ for (a) $\vec{a} \cdot \vec{b}=0$ and (b) $\vec{a} \cdot \vec{b}=\frac{1}{2}$. The colored ellipses indicate the lower bounds of Eq. (6), of allowed values for $\langle A\rangle$ and $\langle B\rangle$ for three different initial degrees of polarization $r$.

blue, dotted red, and dashed orange lines in Fig. 6 for initial degrees of polarization $r_{\text {max }}, r_{\text {mid }}$, and $r_{\text {min }}$, respectively, are reproduced clearly.

\section{Standard deviations}

All states that saturate the state-independent bound of Eq. (8), denoted as $b_{\mathrm{SD}}=1+(\vec{a} \cdot \vec{b})^{2}$, are pure states, found on the surface of the $y-z$ plane on the Bloch sphere. For
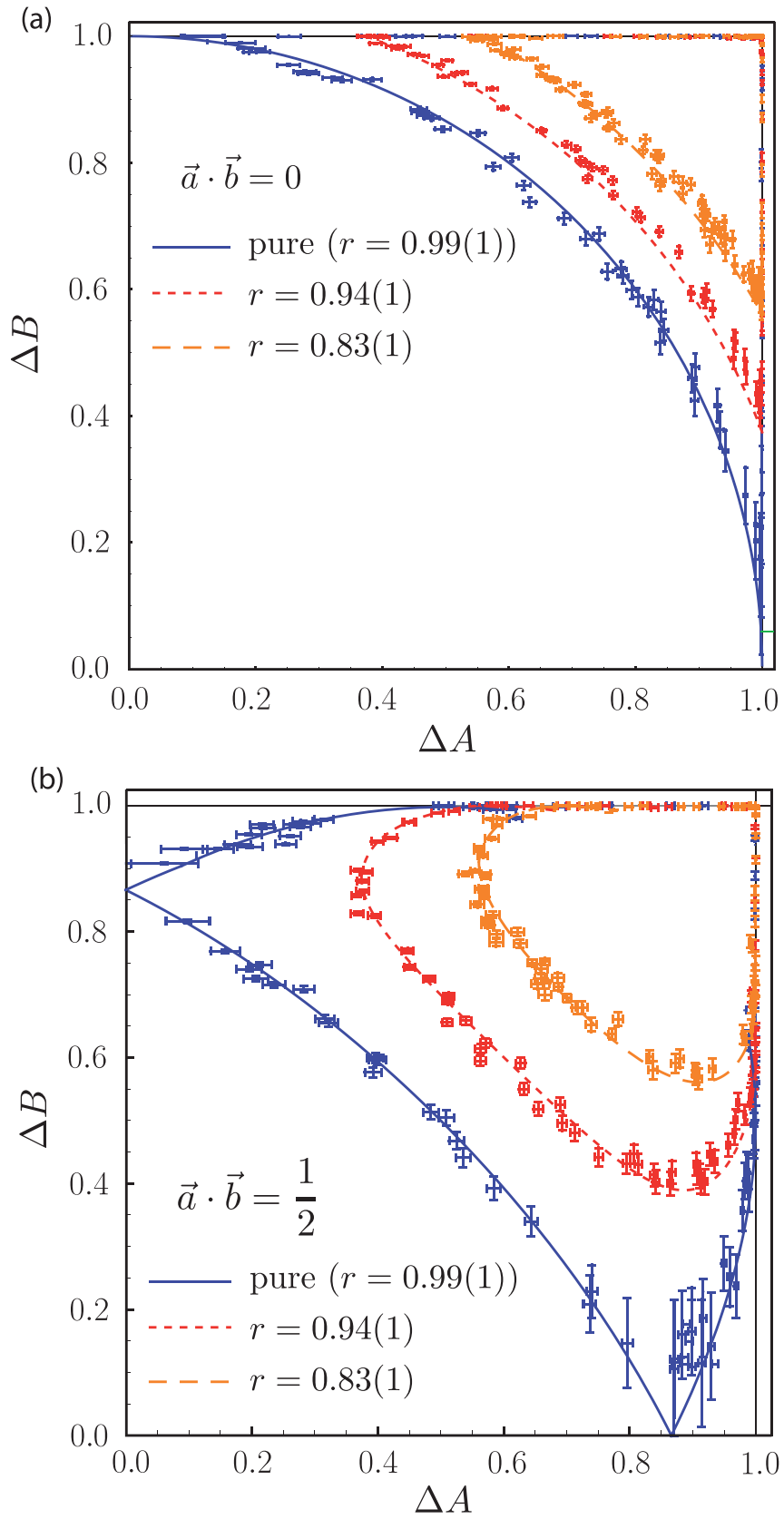

FIG. 7. Plots of the experimentally obtained values for standard deviations $\Delta A$ and $\Delta B$ for (a) $\vec{a} \cdot \vec{b}=0$ and (b) $\vec{a} \cdot \vec{b}=\frac{1}{2}$. The colored curves indicate the lower bounds of Eq. (8) of allowed values for $\Delta A$ and $\Delta B$ for three different initial degrees of polarization $r$.

$\vec{a} \cdot \vec{b}=0$ the partially state-dependent lower bound of Eq. (8), that is $b_{\mathrm{SD}}^{\prime}(r)=2-\left[1-(\vec{a} \cdot \vec{b})^{2}\right]|\vec{r}|^{2}$, is saturated by the corresponding mixed states in the $y-z$ plane of the Bloch sphere with polar angle $\theta \in[0, \pi / 2]$. Unlike the case of expectation values, the lower bound of Eq. (8) is not a closed curve, which can be seen Fig. 7. While initial states $\rho_{\text {in }}^{\theta}$, that lie in the $y-z$ plane, cover the entire the lower bound of Eq. (6), they are insufficient to enclose the remaining boundaries (vertical and horizontal lines in Fig. 7) of allowed values for standard deviations $\Delta A$ and $\Delta B$. For standard deviations, the situation is different compared to expectation values; the vertical and 

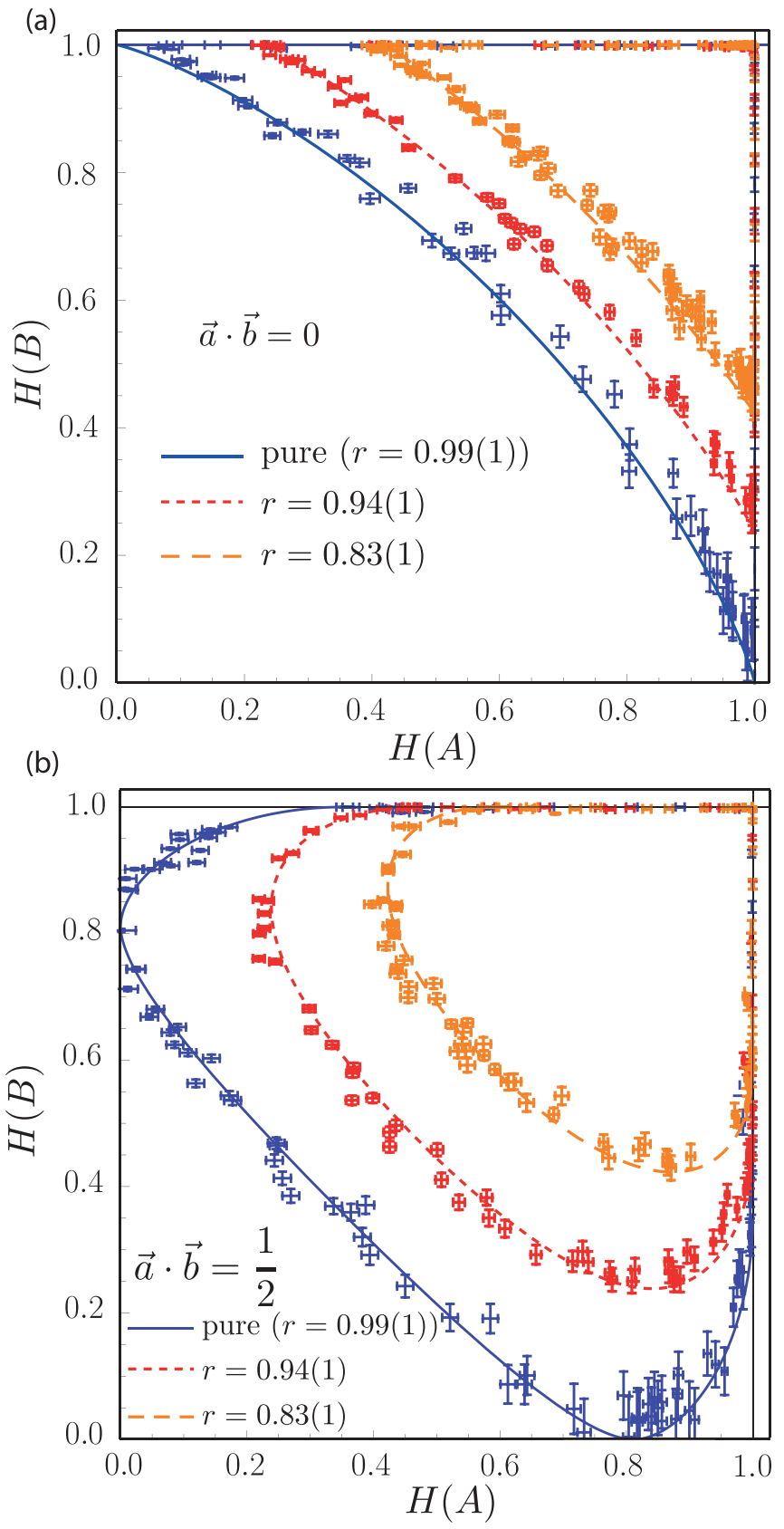

FIG. 8. Plots of the experimentally obtained values for entropies $H(A)$ and $H(B)$ for (a) $\vec{a} \cdot \vec{b}=0$ and (b) $\vec{a} \cdot \vec{b}=\frac{1}{2}$. The colored curves indicate the lower bounds of Eq. (11) of allowed values for $H(A)$ and $H(B)$ for three different initial degrees of polarization $r$.

horizontal boundaries can be saturated not only by pure states (which cover again the entire bound), but (partially) also by certain mixed states $(r<1)$. The vertical and horizontal boundaries of allowed values are occupied by initial states of all mixing angles. For $\vec{a} \cdot \vec{b}=\frac{1}{2}$, depicted in Fig. 7(b), the partially state-dependent lower bounds of Eq. (8) (curved boundaries), are obtained for pure and mixed initial states $\rho_{\text {in }}^{\theta}$ with $\theta \in\left[-\frac{\pi}{6}, \frac{\pi}{2}\right]$, which are randomly generated. For all three initial degrees of polarization $\left(r_{\min }=0.83, r_{\text {mid }}=\right.$ 0.94 , and $r_{\max }=0.99$ ) the theoretical predictions of the tight state-independent and tight partially state-dependent uncer- tainty relations in terms of standard deviations $\Delta A$ and $\Delta B$ (solid blue, dotted red, and dashed orange lines in Fig. 7) are experimentally confirmed.

\section{Entropy}

The obtained results for three initial degrees of polarization $\left(r_{\min }=0.83, r_{\text {mid }}=0.94\right.$, and $\left.r_{\max }=0.99\right)$ are depicted in Fig. 8. Again, as in the case of standard deviations for $\vec{a} \cdot \vec{b}=0$, depicted in Fig. 7(a), all states that saturate the stateindependent bound of Eq. (11), denoted as $b_{H}=1-(\vec{a} \cdot \vec{b})^{2}$, are pure states, located on the surface of the $y-z$ plane on the Bloch sphere with polar angle $\theta \in\left[0, \frac{\pi}{2}\right]$. The partially statedependent lower bound of Eq. (11), expressed as $b_{H}^{\prime}(r)=$ $\left[1-(\vec{a} \cdot \vec{b})^{2}\right]|\vec{r}|^{2}$, is saturated by the corresponding mixed states in the $y-z$ plane of the Bloch sphere with polar angle $\theta \in\left[0, \frac{\pi}{2}\right]$. For $\vec{a} \cdot \vec{b}=\frac{1}{2}$, depicted in Fig. 8(b), the bounds of Eq. (11) are obtained for pure and mixed initial states $\rho_{\text {in }}^{\theta}$ with $\theta \in\left[-\frac{\pi}{6}, \frac{\pi}{3}\right]$. The theoretical predictions (solid blue, dotted red, and dashed orange lines in Fig. 8) are reproduced clearly, demonstrating tight state-independent and tight partially state-dependent uncertainty relations for entropies $H(A)$ and $H(B)$.

\section{DISCUSSION AND CONCLUSION}

The presented experiment investigates the relationship between the expectation values of Pauli spin observables and two standard measures of uncertainty, namely standard deviations and Shannon entropies. The tightness of stateindependent uncertainty relations for Pauli measurements on qubits is experimentally demonstrated. In addition, we observed bounds on these relations, expressed in terms of the norm $|\vec{r}|$ of the Bloch vector, resulting in (partially) statedependent uncertainty relations with lower bounds. We have experimentally confirmed the tightness of state-independent, as well as partially state-dependent, uncertainty relations for pairs of Pauli measurements on qubits. The observed uncertainty relations, expressed in terms of standard deviations and Shannon entropy (both functions of the expectation value), completely characterize the allowed values of uncertainties for Pauli spin observables. The theoretical framework allows for uncertainty relations for three (or more) observables, which will be a topic of forthcoming publications. Finally, we want to emphasize that it is also possible to go beyond projective measurements and give similar relations for positive-operator-valued measures (POVMs) for qubits with binary outcomes, which will be investigated in upcoming experiments.

\section{ACKNOWLEDGMENTS}

The authors thank Alastair A. Abbott and Cyril Branciard for helpful discussions. S.S. and Y.H. acknowledge support by the Austrian Science Fund (FWF) Projects No. P30677 and No. P27666. Y.H. is partly supported by KAKENHI. 
[1] E. H. Kennard, Z. Phys. 44, 326 (1927).

[2] W. Heisenberg, Z. Phys. 43, 172 (1927).

[3] H. P. Robertson, Phys. Rev. 34, 163 (1929).

[4] E. Schrödinger, Sitzungsber. Preuss. Akad. Wiss. Phys. Math. Kl. 14, 296 (1930).

[5] M. Iinuma, Y. Suzuki, T. Nii, R. Kinoshita, and H. F. Hofmann, Phys. Rev. A 93, 032104 (2016).

[6] M. Ozawa, Phys. Rev. A 67, 042105 (2003).

[7] P. Busch, P. Lahti, and R. F. Werner, Phys. Rev. Lett. 111, 160405 (2013).

[8] C. Branciard, Proc. Natl. Acad. Sci. U.S.A. 17, 6742 (2013).

[9] M. Ozawa, arXiv:1404.3388.

[10] J. Erhart, S. Sponar, G. Sulyok, G. Badurek, M. Ozawa, and Y. Hasegawa, Nat. Phys. 8, 185 (2012).

[11] G. Sulyok, S. Sponar, J. Erhart, G. Badurek, M. Ozawa, and Y. Hasegawa, Phys. Rev. A 88, 022110 (2013).

[12] B. Demirel, S. Sponar, G. Sulyok, M. Ozawa, and Y. Hasegawa, Phys. Rev. Lett. 117, 140402 (2016).

[13] G. Sulyok and S. Sponar, Phys. Rev. A 96, 022137 (2017).

[14] B. Demirel, S. Sponar, A. A. Abbott, C. Branciard, and Y. Hasegawa, New J. Phys. 21, 013038 (2019).

[15] B. Demirel, S. Sponar, and Y. Hasegawa, Appl. Sci. 10, 1087 (2020).

[16] L. A. Rozema, A. Darabi, D. H. Mahler, A. Hayat, Y. Soudagar, and A. M. Steinberg, Phys. Rev. Lett. 109, 100404 (2012).

[17] S.-Y. Baek, F. Kaneda, M. Ozawa, and K. Edamatsu, Sci. Rep. 3, 2221 (2013).

[18] F. Kaneda, S.-Y. Baek, M. Ozawa, and K. Edamatsu, Phys. Rev. Lett. 112, 020402 (2014).

[19] M. Ringbauer, D. N. Biggerstaff, M. A. Broome, A. Fedrizzi, C. Branciard, and A. G. White, Phys. Rev. Lett. 112, 020401 (2014).

[20] W. Ma, Z. Ma, H. Wang, Z. Chen, Y. Liu, F. Kong, Z. Li, X. Peng, M. Shi, F. Shi, S.-M. Fei, and J. Du, Phys. Rev. Lett. 116, 160405 (2016).

[21] Y.-L. Mao, Z.-H. Ma, R.-B. Jin, Q.-C. Sun, S.-M. Fei, Q. Zhang, J. Fan, and J.-W. Pan, Phys. Rev. Lett. 122, 090404 (2019).

[22] M. Ozawa, J. Math. Phys. 25, 79 (1984).
[23] P. Busch, P. Lahti, and R. F. Werner, Rev. Mod. Phys. 86, 1261 (2014).

[24] L. A. Rozema, D. H. Mahler, A. Hayat, and A. M. Steinberg, Quantum Stud.: Math. Found. 2, 17 (2015).

[25] M. Ozawa, Phys. Lett. A 318, 21 (2003).

[26] M. J. W. Hall, Phys. Rev. A 69, 052113 (2004).

[27] P. Busch, P. Lahti, and R. F. Werner, Phys. Rev. A 89, 012129 (2014).

[28] F. Buscemi, M. J. W. Hall, M. Ozawa, and M. M. Wilde, Phys. Rev. Lett. 112, 050401 (2014).

[29] G. Sulyok, S. Sponar, B. Demirel, F. Buscemi, M. J. W. Hall, M. Ozawa, and Y. Hasegawa, Phys. Rev. Lett. 115, 030401 (2015).

[30] A. Barchielli, M. Gregoratti, and A. Toigo, Commun. Math. Phys. 357, 1253 (2018).

[31] D. Deutsch, Phys. Rev. Lett. 50, 631 (1983).

[32] H. Maassen and J. B. M. Uffink, Phys. Rev. Lett. 60, 1103 (1988).

[33] K. Kraus, Phys. Rev. D 35, 3070 (1987).

[34] A. Garrett and S. Gull, Phys. Lett. A 151, 453 (1990).

[35] J. Sánches-Ruiz, Phys. Lett. A 244, 189 (1998).

[36] G. Ghirardi, L. Marinatto, and R. Romano, Phys. Lett. A 317, 32 (2003).

[37] J. I. de Vicente and J. Sánchez-Ruiz, Phys. Rev. A 77, 042110 (2008)

[38] M. Berta, M. Christandl, R. Colbeck, J. M. Renes, and R. Renner, Nat. Phys. 6, 659 (2010).

[39] A. K. Pati, M. M. Wilde, A. R. U. Devi, A. K. Rajagopal, and Sudha, Phys. Rev. A 86, 042105 (2012).

[40] H. F. Hofmann and S. Takeuchi, Phys. Rev. A 68, 032103 (2003).

[41] O. Gühne and M. Lewenstein, Phys. Rev. A 70, 022316 (2004).

[42] R. Schwonnek, L. Dammeier, and R. F. Werner, Phys. Rev. Lett. 119, 170404 (2017).

[43] A. A. Abbott, P.-L. Alzieu, M. J. W. Hall, and C. Branciard, Mathematics 4, 8 (2016).

[44] P. Giorda, L. Maccone, and A. Riccardi, Phys. Rev. A 99, 052121 (2019). 